\title{
Antibodies against Desmoglein 3 (Pemphigus Vulgaris Antigen) Are Present in Sera from Patients with Paraneoplastic Pemphigus and Cause Acantholysis In Vivo in Neonatal Mice
}

\author{
Masayuki Amagai, ${ }^{*}$ Takeji Nishikawa, ${ }^{\star}$ Hossein C. Nousari, ${ }^{\ddagger}$ Grant J. Anhalt, ${ }^{\ddagger}$ and Takashi Hashimoto ${ }^{\S}$ \\ *Department of Dermatology, Keio University School of Medicine, Tokyo 160-8582, Japan; ${ }^{\ddagger}$ Department of Dermatology, Johns Hopkins \\ University, Baltimore, Maryland 21205; and ${ }^{\S}$ Department of Dermatology, Kurume University School of Medicine, Fukuoka 830-0011, Japan
}

\begin{abstract}
Paraneoplastic pemphigus (PNP) is an autoimmune blistering disease that occurs in association with underlying neoplasms. Patients with PNP develop characteristic IgG autoantibodies directed against multiple antigens, most of which have been identified as cytoplasmic proteins of the plakin family (desmoplakin I, II, BPAG1, envoplakin, and periplakin). This study identified cell surface target antigens of PNP. We focused on desmoglein (Dsg) 3 and Dsg1, the autoantigens of pemphigus vulgaris and pemphigus foliaceus. ELISA using baculovirus-expressed recombinant Dsgs (rDsg3, rDsg1) has revealed that 25 out of 25 PNP sera tested were positive against Dsg3 and 16 of 25 were positive against Dsg1. All of 12 PNP sera tested immunoprecipitated Dsg3. Removal of anti-Dsg3 autoantibodies by immunoadsorption was sufficient to eliminate the ability of PNP sera to induce cutaneous blisters in neonatal mice in vivo. Furthermore, anti-Dsg3-specific antibodies that were affinity purified from PNP sera were proven to be pathogenic and caused blisters in neonatal mice. These findings indicate that Dsg3 and Dsg1 are the cell surface target antigens in PNP and that IgG autoantibodies against Dsg3 in PNP sera play a pathogenic role in inducing loss of cell adhesion of keratinocytes and causing blister formation. (J. Clin. Invest. 1998. 102:775-782.) Key words: cadherin • neoplasm • autoimmunity • desmosomes • ELISA
\end{abstract}

\section{Introduction}

Pemphigus is a group of acquired autoimmune blistering diseases of the skin and mucous membranes that is characterized histologically by intraepidermal blisters caused by the separation of epidermal cells (acantholysis) and immunopathologically by the discovery of in vivo bound and circulating IgG di-

Part of this work was presented at the International Investigative Dermatology Meeting in Cologne, Germany, 7-10 May 1998 and published in abstract form (1998. J. Invest. Dermatol. 110:482a).

Address correspondence to Masayuki Amagai, M.D., Ph.D., Department of Dermatology, Keio University School of Medicine, 35 Shinanomachi, Shinjuku-ku, Tokyo 160, Japan. Phone: 81-3-33531211 Ext. 2414; FAX: 81-3-3351-6880; E-mail: amagai@mc.med.keio. ac.jp

Received for publication 7 April 1998 and accepted in revised form 5 June 1998

J. Clin. Invest.

(C) The American Society for Clinical Investigation, Inc. 0021-9738/98/08/0775/08 \$2.00

Volume 102, Number 4, August 1998, 775-782

http://www.jci.org rected against the cell surface of keratinocytes (1). Classic pemphigus includes two major subtypes, pemphigus vulgaris $(\mathrm{PV})^{1}$ and pemphigus foliaceus $(\mathrm{PF})$. Autoimmune targets in these forms of pemphigus are desmoglein 3 (Dsg3) and Dsg1, respectively $(2,3)$. The desmogleins are members of the cadherin family of cell-cell adhesion molecules in desmosomes.

Paraneoplastic pemphigus (PNP) is a recently described form of pemphigus that occurs in association with underlying neoplasms $(4,5)$. PNP is unique and distinct from the classic forms of PV and PF by clinical, histologic, and immunopathologic criteria. PNP is defined by the following five criteria: $(a)$ the presence of mucosal ulcerations and blisters and a polymorphous skin eruption in the context of an occult or known neoplasm; (b) histologic findings of vacuolar interface change, keratinocyte necrosis, and intraepidermal cell-cell detachment (suprabasilar acantholysis); (c) deposition of IgG and C3 on epidermal cell surfaces and variably also along the basement membrane zone; $(d)$ serum autoantibodies that bind to the cell surface of stratified squamous epithelia and also to simple, columnar, and transitional epithelia; and (e) serum autoantibodies that recognize a characteristic antigen complex of 250-, 230-, 210-, 190-, and 170-kD peptides by immunochemical techniques such as immunoprecipitation. Not all individuals who have this disorder fulfill completely each one of these criteria. The standard for diagnosis of PNP at present is identification of the characteristic antigen complex by serum autoantibodies, as shown by immunoprecipitation. Neoplasms most commonly associated with PNP are non-Hodgkin's lymphoma, chronic lymphocytic leukemia, Castleman's disease, thymomas, and poorly differentiated spindle-cell sarcomas.

The molecules of PNP antigen complex have been mostly identified. The 250- and 210-kD antigen were identified as desmoplakin I and II, which are the major cytoplasmic plaque proteins of desmosomes $(4,6)$. The $230-\mathrm{kD}$ antigen is BPAG1, the major plaque protein of the epidermal hemidesmosome and one of the target antigens of bullous pemphigoid $(4,7)$. Subsequent immunoblot studies have revealed that the 210 and $190-\mathrm{kD}$ antigens were most consistently recognized by PNP sera. The 210-kD antigen recognized by immunoprecipitation was found to be a doublet, of which the slower migrating band was desmoplakin II. The faster migrating band, which is recognized by immunoblotting (8), was later identified by cDNA cloning as envoplakin (9). Envoplakin is a novel precursor protein of the cornified envelope that is homologous to desmoplakin (10). Recently, the 190-kD antigen was identified as periplakin (11) (Kiyokawa, C., C. Ruhrberg, T. Karashima, O. Mori, T. Nishikawa, K.J. Green, G.J. Anhalt, F.M. Watt,

1. Abbreviations used in this paper: Dsg, desmoglein; $\mathrm{PF}$, pemphigus foliaceus; PNP, paraneoplastic pemphigus; PV, pemphigus vulgaris; rDsg, recombinant desmoglein. 
and T. Hashimoto, manuscript submitted for publication), which is also homologous to desmoplakin (12). The $170-\mathrm{kD}$ antigen is a transmembrane cell surface protein, which is yet to be identified (5). To date, there has been only a suggestion that Dsg3, which is a desmosomal cadherin and the target antigen of $\mathrm{PV}$, is recognized by a small number of PNP sera $(8,13,14)$.

Among the identified antigens, desmoplakin I, II, BPAG1, envoplakin, and periplakin are five proteins that have related sequences, and all are members of the plakin gene family (15). However, all of these plakin proteins are located in the cytoplasm of the keratinocytes, and presumably, circulating autoantibodies cannot access them. Therefore, it is hard to envision how these antigens can be directly involved in the induction of loss of cell-cell adhesion of keratinocytes.

We have previously produced recombinant Dsgs (rDsgs) by using a baculovirus expression system, which express most, if not all, conformational epitopes of the native antigens that are critical in the pathogenesis of the clinical manifestations of pemphigus $(16,17)$. Using these rDsgs, we have developed an ELISA as a sensitive and highly specific assay for the detection of autoantibodies against Dsgs (18).

In this study, we have attempted to identify molecules that are targeted by pathogenic autoantibodies in PNP sera to induce blister formation. We focused on immunoreactivity of PNP sera against Dsg3 and Dsg1 because these are the target molecules of the classic pemphigus and appear to be recognized by a subset of sera from PNP patients. Our studies demonstrated that Dsg3 is a major target cell surface molecule in PNP and that anti-Dsg3 IgG plays a primary pathogenic role in inducing loss of cell adhesion of keratinocytes and causing blister formation in PNP.

\section{Methods}

Human sera. Sera were obtained from 25 patients with clinically, histologically, immunochemically, and immunohistochemically typical PNP. Six of these were obtained by plasmapheresis. Sera were obtained from 21 patients with typical PV, and 31 normal individuals as controls.

Production of $r$ Dsg in baculovirus expression. We previously produced chimeric molecules that contain the entire extracellular domain of Dsg3 or Dsg1 fused with the constant region of human IgG1 $(16,17)$. To improve purification steps, a His-tag was added to the $\mathrm{C}$ termini of the proteins. cDNA encoding the constant region of human IgG1 and His-tag was amplified on pEVmod-PVIg by PCR with the following primers: 5'-primer, 5'-GGCCTCGAGCCCAAATCTTGTGAC-3', and $3^{\prime}$-primer, 5'-CGGGGTACCTCAATGATGATGATGATGATGTTTACCCGGGGACAGGGAGAG-3'. The PCR product was digested with $\mathrm{XhoI}$ and $\mathrm{KpnI}$ and ligated with XhoIKpnI-cut pEVmod-PVIg. This construct was designated pEVmodDsg3-Ig-His. cDNA encoding the extracellular domain of Dsg1 was obtained by PCR, as previously reported (17), and used in place of the Dsg3 extracellular domain of pEVmod-Dsg3-Ig-His by digestion with BglII and XhoI. This construct was designated pEVmod-Dsg1Ig-His.

Recombinant baculoviruses were obtained by cotransfection of pEVmod-Dsg3-Ig-His or pEVmod-Dsg1-Ig-His with BaculoGold baculovirus DNA (Pharmingen, San Diego, CA) into cultured insect Sf9 cells. High Five cells (Invitrogen, San Diego, CA) cultured in serum-free EX Cell 405 medium (JRH Bioscience, Lenexa, KS) were infected with the recombinant viruses and incubated for $3 \mathrm{~d}$. Recombinant baculoproteins $\mathrm{rDsg} 3$-Ig-His and $\mathrm{rDsg} 1$-Ig-His were produced in the culture supernatant. Supernatants were stored at $-70^{\circ} \mathrm{C}$ after removal of cell debris by centrifugation. These baculoproteins were purified and concentrated on TALON (Clontech, Palo Alto, CA) affinity metal resin by mixing with the culture supernatants at $4^{\circ} \mathrm{C}$ overnight. The molecular masses of $\mathrm{rDsg} 3-\mathrm{Ig}-\mathrm{His}$ and $\mathrm{rDsg} 1-\mathrm{Ig}$-His were 107 and $112 \mathrm{kD}$, respectively, as detected by immunoblotting with anti-human $\mathrm{IgG}$ antibodies.

ELISA against Dsg3 and Dsg1. ELISA scores against Dsg3 and Dsg1 were obtained with slight modification as previously described (18). ELISA plates coated with rDsg3-His or rDsg1-His were incubated for $1 \mathrm{~h}$ at room temperature with sera diluted 201-fold with dilution buffer ( $1 \% \mathrm{BSA}, 1 \mathrm{mM} \mathrm{CaCl}$, and $0.05 \%$ Tween 20 in Trisbuffered saline, $\mathrm{pH}$ 7.4), washed, and then incubated with 1,000-folddiluted peroxidase-conjugated mouse anti-human IgG mAb (MBL, Nagoya, Japan) for $1 \mathrm{~h}$. After washing, color development was achieved with $1.6 \mathrm{mM}$ tetramethylbenzidine (Sigma Chemical Co., St. Louis, MO) in $10 \mathrm{mM}$ sodium citrate, $1.25 \%$ polyethylene glycol 4000 , and $10 \mathrm{mM}$ hydrogen peroxide and terminated with $2 \mathrm{~N} \mathrm{H}_{2} \mathrm{SO}_{4}$. Single PV and PF sera were selected as the standard for each respective ELISA. Index value was defined as follows: index value $=$ (ODsample - ODnegative $) /($ ODpositive - ODnegative $) \times 100$. A cutoff value was defined as the average value +3 SD of normal control sera (9.4 for Dsg3 ELISA, 9.1 for Dsg1 ELISA). Each sample was run in duplicate.

Immunoadsorption and elution of PNP sera with $r D s g$. rDsg3-IgHis and rDsg1-Ig-His baculoproteins were purified on TALON metal affinity resin by incubating with culture supernatants at $4^{\circ} \mathrm{C}$ overnight. After washing the resin with PBS with $1 \mathrm{mM} \mathrm{CaCl}_{2}$ (PBS-Ca), we incubated PNP sera diluted twofold with PBS, with the resin at $4^{\circ} \mathrm{C}$ overnight with gentle rocking. The removal of respective autoantibodies was confirmed by ELISA against Dsg3 and Dsg1. Then, IgG fraction was prepared by precipitation with $40 \%$ ammonium sulfate, dialyzed against PBS, and concentrated $\sim 10$-fold with the microconcentrator Centriprep 30 (Millipore-Amicon, Beverly, MA).

IgG bound to rDsg3-Ig-His or the resin itself was eluted off with IgG elution buffer (Pierce Chemical Co., Rockford, IL), immediately neutralized with Tris- $\mathrm{HCl}, \mathrm{pH} 7.4$, dialyzed against PBS, and concentrated with Centriprep 30. Specificity of eluted IgG was tested by ELISA.

Immunofluorescence. Normal human skin sections and rat urinary bladder sections were used as substrates for immunofluorescence staining.

Immunoblot and immunoprecipitation analyses. Immunoblot analysis of extracts of EDTA-separated normal human epidermis was performed as described previously $(8,19)$. Immunoprecipitation was performed according to the technique described previously using cultured normal human keratinocytes that were radiolabeled with $\left[{ }^{14} \mathrm{C}\right]$ amino acids and extracted with extraction buffer containing Nonidet P-40 (6).

To determine whether PNP sera contain IgG against Dsg3, a combined immunoprecipitation and immunoblot was performed using insect cell culture supernatant containing rDsg3-His (18) or keratinocyte extracts as a substrate. The keratinocyte extracts were prepared from KU8 cells, a squamous carcinoma cell line (20), with $1 \%$ Triton X-100 in PBS containing complete protease inhibitor cocktail tablet (Boehringer Mannheim, Mannheim, Germany). The rDsg3-His culture supernatant or the KU8 extracts were incubated with PNP, $\mathrm{PV}$, or normal control sera at $4^{\circ} \mathrm{C}$ for $2 \mathrm{~h}$, then immunoprecipitated with protein A Sepharose CL4B (Pharmacia Biotech, Uppsala, Sweden). The immunoprecipitants were applied to SDS-PAGE and blotted to a PVDF membrane (Millipore Corp., Bedford, MA). Dsg3 was visualized with a mouse anti-human Dsg3 monoclonal antibody 5H10 (Proby, C., T. Ohta, K. Nishifuji, M.J. Wheelock, H. Suzuki, S. Koyasu, S. Gamou, N. Shimizu, T. Nishikawa, and M. Amagai, manuscript in preparation).

Neonatal mouse study. To evaluate the pathogenic activity of immunoadsorbed PNP sera and affinity-purified IgG on rDsg3-Ig-His, we used neonatal mouse model for pemphigus, as previously described $(16,17)$. Neonatal ICR mice were obtained at $12-24 \mathrm{~h}$ of age (body weight, 1.5-2.0 g). The enriched IgG solution prepared from the immunoadsorbed PNP sera was injected subcutaneously at $\sim 10$ 
$\mathrm{mg}$ of total protein per gram of body weight. The affinity-purified anti-Dsg3 IgG was injected at $0.2-1 \mathrm{mg}$ of total protein per gram of body weight. The concentration of the total protein was calculated by measuring $\mathrm{OD}_{280}\left(\mathrm{OD}_{280}\right.$ of $\left.1 \mathrm{mg} / \mathrm{ml} \mathrm{IgG}=1.43\right)$. The neonatal mice were examined and biopsied $6-18 \mathrm{~h}$ after injection.

\section{Results}

PNP sera contain autoantibodies against Dsg3 and Dsg1. We have developed ELISAs against rDsg3 and rDsg1 as a specific and sensitive detection tool for anti-Dsg3 and anti-Dsg1 autoantibodies (18). To determine whether PNP sera contain autoantibodies against the antigens of classic pemphigus, Dsg3 or Dsg1, we ran 25 PNP sera on the Dsg3 and Dsg1 ELISAs (Fig. 1). Remarkably, all of 25 PNP sera tested (100\%) exceeded the cutoff value for Dsg3 ELISA, and 16 of them (64\%) also exceeded the cutoff value for Dsg1 ELISA. Out of 21 PV sera that were used as positive controls, all $21(100 \%)$ were positive against Dsg3, and 13 (62\%) were positive against Dsg1. None of 31 normal control sera $(0 \%)$ were positive against either Dsg3 or Dsg1.

Conventional immunoprecipitation using radiolabeled keratinocyte extracts failed to detect the 130-kD Dsg3 (5) (Fig. 3). To improve specificity and sensitivity for detection of Dsg3, we performed an immunoprecipitation-immunoblot assay. We used culture supernatant of insect cells containing rDsg3-His (18) and keratinocyte extracts prepared from KU8 cells as a substrate. All of the 12 PNP sera tested immunoprecipitated rDsg3-His in the culture supernatant as well as the $130-\mathrm{kD}$ Dsg3 in the keratinocyte extracts, which was visualized by immunoblotting with a mouse anti-Dsg3 monoclonal antibody, 5H10 (Fig. 2). All of the five PV sera tested, but none of the 10 normal control sera, detected rDsg3-His and Dsg3.

These findings indicate that essentially all PNP sera contain IgG autoantibodies against Dsg3 and that more than half of PNP sera contain autoantibodies against Dsg1 in addition to anti-Dsg3 antibodies.

Immunoadsorption by $r D s g$ was performed in an antigenspecific way. To determine whether removal of anti-Dsg au- toantibodies from PNP sera alter their pathogenic activity, we immunoadsorbed PNP sera with rDsgs. Six PNP sera, which were obtained by plasmapheresis, were used for the immunoadsorption study. When injected in neonatal mice, IgG fraction caused gross blisters in all of them. Two mice (PNP\#1 and PNP\#2 in Table I) were positive against both Dsg3 and Dsg1 (Dsg3+/Dsg1+), and the other four (PNP\#3 to PNP\#6 in Table I) were positive only against Dsg3 (Dsg3+/Dsg1-). The two Dsg3+/Dsg1+ PNP sera were immunoadsorbed with a mixture of rDsg3-Ig-His and rDsg1-Ig-His or with rDsg3-IgHis alone, and the four Dsg3+/Dsg1- PNP sera were incubated with rDsg3-Ig-His alone. As a negative control of the immunoadsorption, these sera were incubated with only the metal affinity resin. As a control serum, one PV serum was immunoadsorbed in the same way. Index values of Dsg3 and Dsg1 ELISA became negative after the immunoadsorption with the respective rDsg (Table I). However, indirect immunofluorescence staining of rat bladder, which is thought to be the best substrate to detect PNP autoantibodies (21), was not altered or only slightly diminished (data not shown). In addition, the adsorbed sera were examined by immunoprecipitation and compared with unadsorbed sera (Fig. 3). The incubation of the PNP sera with the mixture of rDsg3-Ig-His and rDsg1-Ig-His did not reduce any of the bands of the PNP antigen complex detected by immunoprecipitation. The 130-kD Dsg3 antigen was not identified by any of the sera by this technique. This incubation did not alter the intensity of the $210-\mathrm{kD}$ envoplakin and $190-\mathrm{kD}$ periplakin bands as detected by immunoblot (data not shown).

These observations indicate that the immunoadsorption by rDsgs was performed in an antigen-specific way and that there is no cross-reactivity of anti-Dsg autoantibodies against other proteins in the PNP antigen complex.

Immunoadsorption of PNP sera with $r D s g 3$ eliminated their pathogenic activity to induce blisters in neonatal mice in vivo. IgG fraction was prepared from the sera that were incubated with rDsgs on the resin or with the resin alone, and injected into neonatal mice to examine their pathogenic activity of blister formation in the skin (Table I; Fig. 4). Mice injected with
Dsg3 ELISA

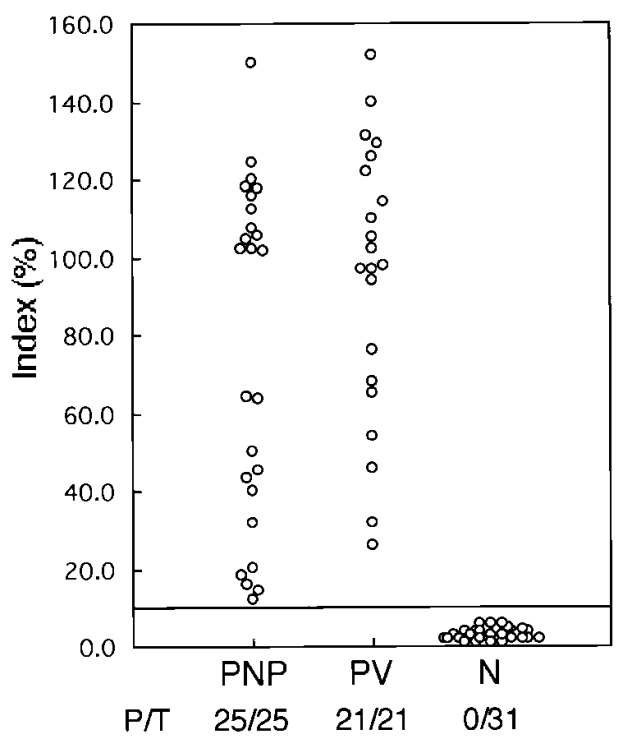

\section{Dsg1 ELISA}

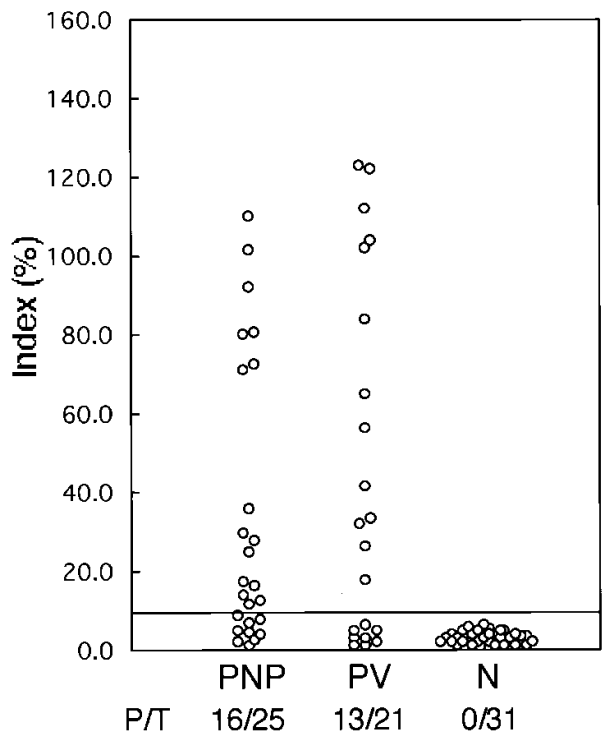

Figure 1. Anti-Dsg3 and anti-Dsg1 IgG titers obtained by ELISA using recombinant Dsg3 and Dsg1. PNP, $\mathrm{PV}$, and normal control sera $(N)$ were examined. $\mathrm{P} / \mathrm{T}$ indicates positive vs. total number examined. All PNP sera were positive against Dsg3, and more than half of PNP sera were positive against Dsg1. The cutoff value was 9.4 for Dsg3 ELISA and 9.1 for Dsg1 ELISA. 


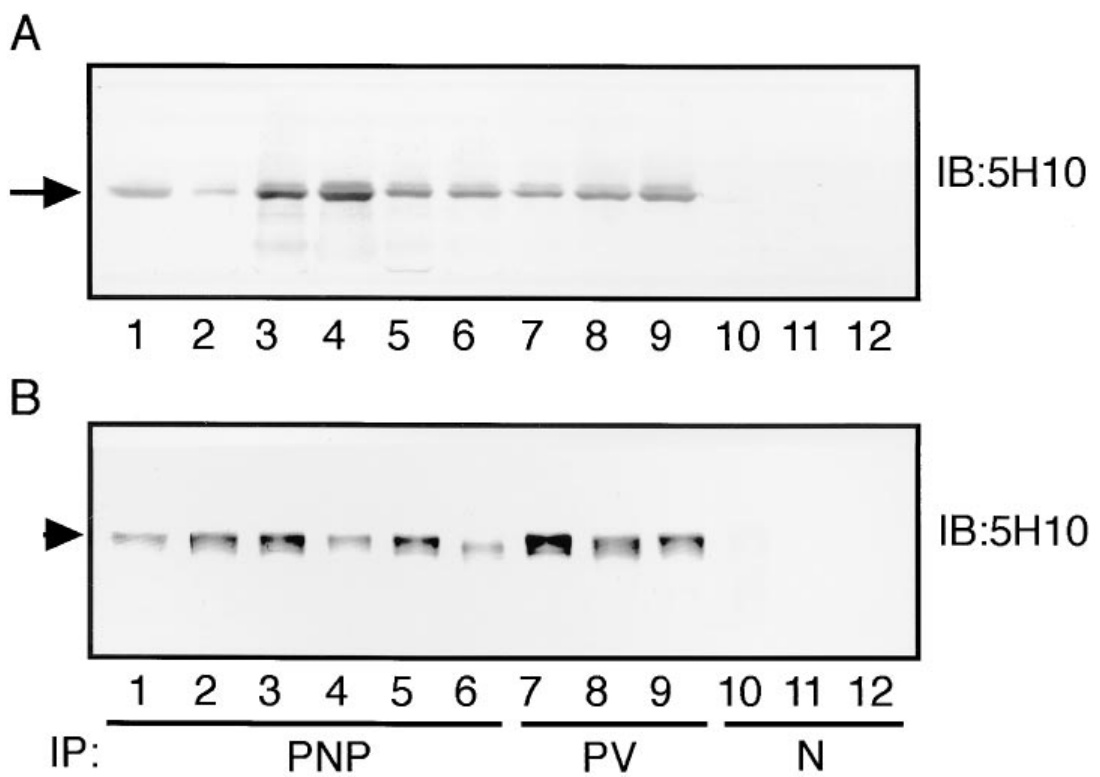

Figure 2. Demonstration of anti-Dsg3 IgG by immunoprecipitation-immunoblot assay. Culture supernatant of insect cells containing rDsg3-His $(A)$ or keratinocyte extract prepared from KU8 cells $(B)$ was incubated with PNP (lanes 1-6), PV (lanes 7-9), or normal control ( $N$, lanes 10-12) sera and immunoprecipitated with protein A Sepharose. Then the precipitated $\mathrm{rDsg} 3$-His $(A)$ or Dsg3 $(B)$ was visualized by immunoblotting with an anti-Dsg3 monoclonal antibody, 5H10. An arrow $(A)$ and an arrowhead $(B)$ indicate the band for $\mathrm{rDsg3}$-His and Dsg3, respectively. All PNP sera immunoprecipitated Dsg3.
IgG prepared from Dsg3+/Dsg1+ PNP sera (PNP\#1 and PNP\#2) after incubation with the resin alone showed extensive blisters and erosions, with loss of cell adhesion of keratinocytes just above the basal cell layers (suprabasilar acantholysis), as previously reported (Fig. $4 A$, left mice, and $B$ ) (4). Direct immunofluorescence of mouse skin showed human $\mathrm{IgG}$ deposition not only on keratinocyte cell surfaces and basement

Table I. The Effects of Immunoadsorption of PNP Sera with rDsg3 and rDsg1

\begin{tabular}{|c|c|c|c|c|c|c|}
\hline \multirow[b]{2}{*}{ Serum } & \multirow[b]{2}{*}{ Immunoadsorption* } & \multicolumn{2}{|c|}{ ELISA } & \multicolumn{3}{|c|}{ Neonatal mice } \\
\hline & & Dsg3 & Dsg1 & $n$ & Blister & $\mathrm{DIF}^{\S}$ \\
\hline \multirow[t]{2}{*}{ PNP\#1 } & None & 140.1 & 54.6 & 4 & ++ & ++ \\
\hline & rDsg3+rDsg1 & 2.0 & 7.4 & 4 & - & ++ \\
\hline \multirow[t]{2}{*}{ PNP\#1 } & None & 136.5 & 50.9 & 3 & ++ & ++ \\
\hline & rDsg3 & 1.0 & 42.3 & 3 & - & ++ \\
\hline \multirow[t]{2}{*}{ PNP\#2 } & None & 118.4 & 75.4 & 4 & ++ & ++ \\
\hline & rDsg3+rDsg1 & 6.1 & 1.6 & 4 & - & ++ \\
\hline \multirow[t]{2}{*}{ PNP\#2 } & None & 124.7 & 63.7 & 3 & ++ & ++ \\
\hline & rDsg3 & 6.3 & 62.1 & 3 & - & ++ \\
\hline \multirow[t]{2}{*}{ PNP\#3 } & None & 104.2 & 0.3 & 4 & + & + \\
\hline & rDsg3 & 0.4 & 0.1 & 4 & - & + \\
\hline \multirow[t]{2}{*}{ PNP\#4 } & None & 98.4 & 2.5 & 4 & + & + \\
\hline & rDsg3 & 0.2 & 0.8 & 4 & - & + \\
\hline \multirow[t]{2}{*}{ PNP\#5 } & None & 105.2 & 1.1 & 4 & + & + \\
\hline & rDsg3 & 1.8 & 1.5 & 4 & - & + \\
\hline \multirow[t]{2}{*}{ PNP\#6 } & None & 68.6 & 3.6 & 3 & + & + \\
\hline & rDsg3 & 2.5 & 3.2 & 3 & - & + \\
\hline \multirow[t]{2}{*}{ PV\#1 } & None & 124.5 & 87.5 & 3 & ++ & ++ \\
\hline & rDsg3+rDsg1 & 2.6 & 3.1 & 3 & - & - \\
\hline
\end{tabular}

*PNP or PV sera were immunoadsorbed with a mixture of rDsg3-Ig-His and rDsg1-Ig-His or rDsg3-Ig-His alone or with the metal resin as a control (None). ${ }^{\S} D I F$, direct immunofluorescence on the skin of mice to determine whether human $\mathrm{IgG}$ deposition is found on keratinocyte cell surfaces. membrane zone but also in the cytoplasm (Fig. $4 \mathrm{D}$ ). In contrast, when the same two Dsg3+/Dsg1+ PNP sera were preincubated with the mixture of $\mathrm{rDsg} 3-\mathrm{Ig}$-His and $\mathrm{rDsg} 1-\mathrm{Ig}-\mathrm{His}$, these $\mathrm{IgG}$ fractions failed to induce gross blisters and erosions in the mice (Fig. $4 \mathrm{~A}$, right mice). Furthermore, histologic analysis of the skin revealed no clear evidence of blister formation (Fig. $4 \mathrm{C}$ ). However, direct immunofluorescence remained

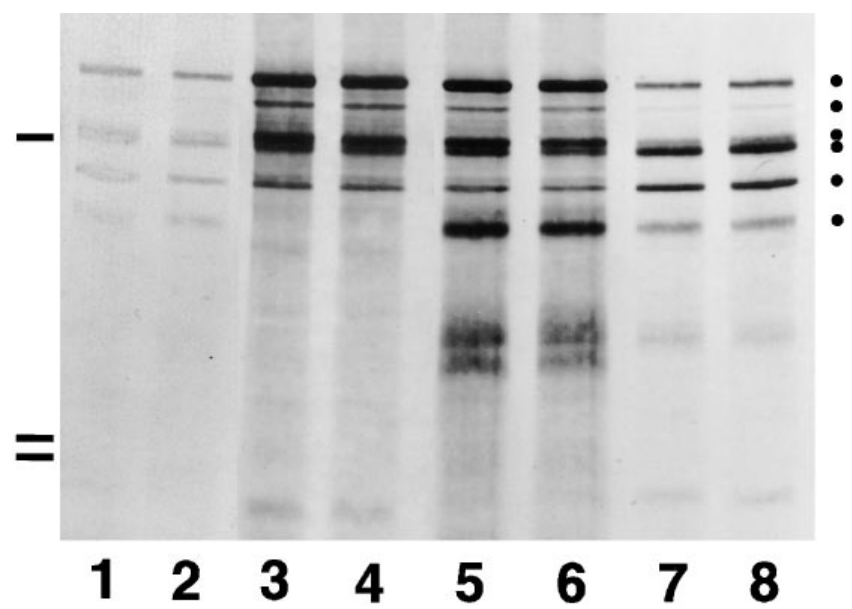

Figure 3. Immunoprecipitation of the PNP antigen complex with PNP sera immunoadsorbed with rDsgs. Four of the PNP sera that were adsorbed with the mixture of rDsg3-Ig-His and rDsg1-Ig-His are shown (lanes 2, 4,6, and 8 ) and compared with unadsorbed serum from which they were obtained (lanes $1,3,5$, and 7 ). These sera also appeared in Table I ( $P N P \# 1$, lanes 5 and $6 ; P N P \# 2$, lanes 7 and 8 ; $P N P \# 3$, lanes 3 and 4 ; and $P N P \# 5$, lanes 1 and 2). There is no difference in the intensity of the bands present in the adsorbed vs. the unadsorbed samples. The $130-\mathrm{kD}$ Dsg3 antigen was not identified by any of the sera by this technique. Closed circles on the right side indicate the positions of the PNP antigen complex; from top to bottom, $250 \mathrm{kD}$ (desmoplakin I), $230 \mathrm{kD}$ (BPAG1), 210a kD (desmoplakin II), 210b kD (envoplakin), $190 \mathrm{kD}$ (periplakin), and $170 \mathrm{kD}$ (unidentified). The bars on the left side indicate molecular mass standards at 200, 116, and $97 \mathrm{kD}$. 

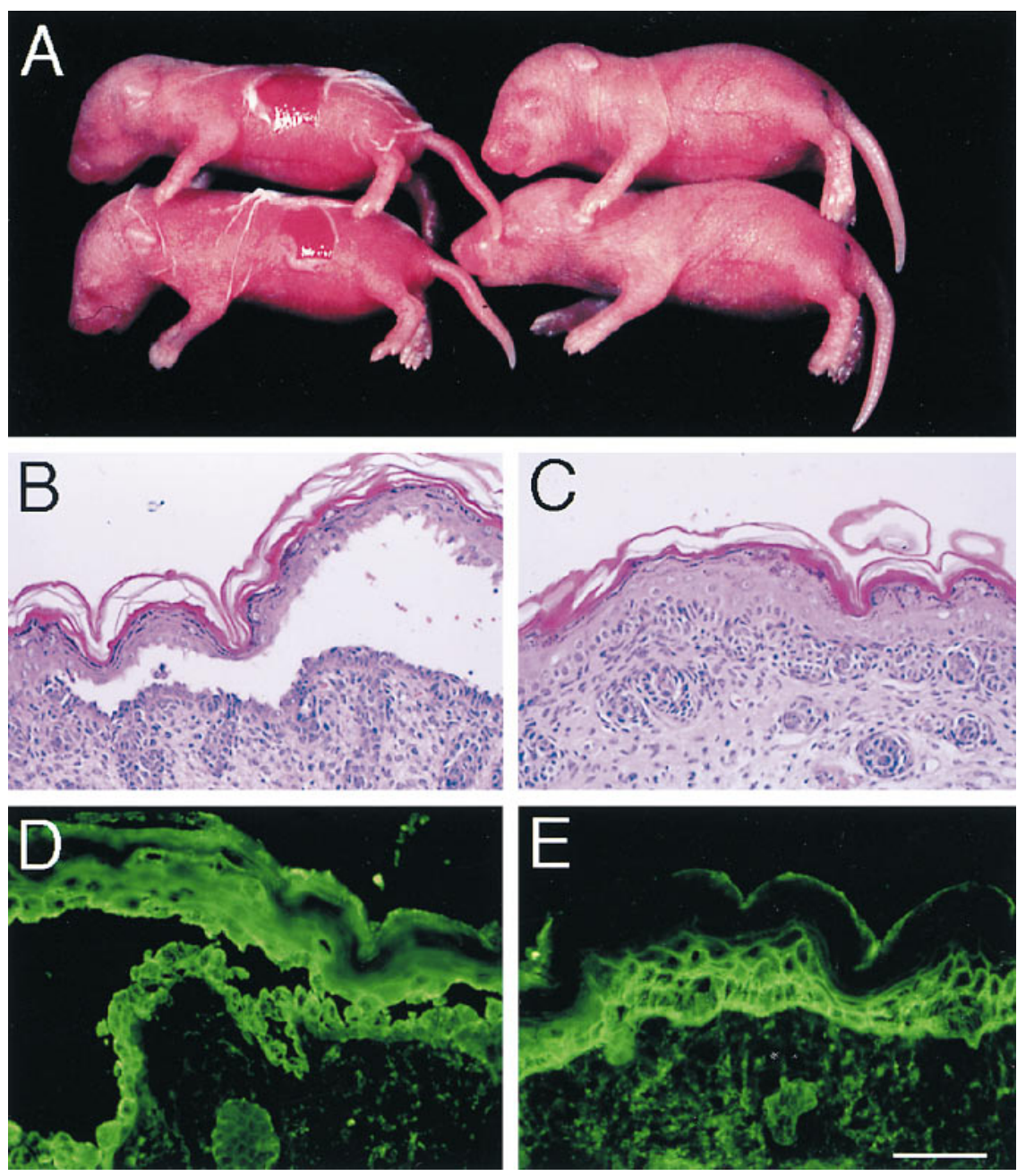

Figure 4. Removal of anti-Dsg3 and anti-Dsg1 IgG eliminates the pathogenic activity of PNP sera. A PNP serum (PNP\#1 in Table I) was incubated either with the mixture of rDsg3Ig-His and rDsg1-Ig-His or with the metal resin alone; IgG then was prepared, concentrated, and injected into neonatal mice. Mice injected with $\mathrm{IgG}$ from PNP sera after incubation with the control resin demonstrated extensive blisters and erosions ( $A$, left two mice) and showed suprabasilar acantholysis in histology $(B)$. Human IgG deposition was found not only on keratinocyte cell surfaces and basement membrane zone but also in the cytoplasm $(D)$. In contrast, mice injected with IgG from PNP sera after incubation with the mixture of rDsg3-IgHis and $\mathrm{rDsg} 1-\mathrm{Ig}-\mathrm{His}$ showed intact skin $(A$, right two mice) with no clear evidence of blister formation $(C)$. However, deposition of human $\mathrm{IgG}$ was noted on keratinocyte cell surfaces and basement membrane zone $(E)$. positive for human IgG on keratinocyte cell surfaces and basement membrane zone (Fig. 4 E). IgG was prepared in the same way from these Dsg3+/Dsg1+ PNP sera after immunoadsorption with Dsg3-Ig-His alone. This immunoadsorbed IgG failed to induce blisters or erosions (Table I). Furthermore, IgG prepared from the four Dsg3+/Dsg1- PNP sera (PNP\#3, 4, 5, 6 in Table I) after immunoadsorption with Dsg3-Ig-His alone also failed to induce blister formation in neonatal mice in vivo.

These findings indicate that the removal of anti-Dsg3 autoantibodies is sufficient to eliminate the pathogenic activity of PNP sera and to prevent blister formation in neonatal mice in vivo.

Anti-Dsg3-specific IgG affinity purified from PNP sera caused acantholysis in neonatal mice. To further determine whether anti-Dsg3 autoantibodies in PNP sera are pathogenic enough to induce blister formation by themselves, the antibodies were affinity purified on rDsg3-Ig-His from PNP\#1 serum and injected into neonatal mice. As a control, PNP sera were incubated with resin alone, and eluates from the resin were prepared and injected in an identical fashion. The specificity of the affinity-purified IgG was confirmed by ELISA (index values: Dsg3 ELISA, 145.3; Dsg1 ELISA, 3.8). These anti-Dsg3specific antibodies resulted in the formation of gross blisters in neonatal mice $(n=5)$ (Fig. $5 \mathrm{~A}$, left mouse), although the extent of blisters was not as severe as that of mice injected with the whole IgG of PNP\#1 serum. Histologic analysis of the skin revealed suprabasilar acantholysis (Fig. 5 B), as found in the mice injected with PNP sera. The mice showed positive human IgG deposition on the keratinocyte cell surfaces only in the lower epidermis (Fig. $5 \mathrm{D}$ ). The antibodies eluted from the control resin showed no reactivity against Dsgs by ELISA (Dsg3, 2.3; Dsg1, 1.8) and did not show any sign of blister formation with no human IgG deposition $(n=4)$ (Fig. $5 \mathrm{~A}$, right mouse, $C$ and $E$ ). Anti-Dsg3 antibodies affinity purified from the PNP\#2 serum showed similar results (data not shown).

These findings indicate that anti-Dsg3 IgG autoantibodies in PNP sera are pathogenic and can induce loss of cell-cell keratinocyte adhesion in neonatal mice in vivo.

\section{Discussion}

PNP is a unique form of pemphigus that is associated with underlying neoplasms and is clinically, histologically, and immunopathologically distinct from PV and PF. To date, antigens recognized by the autoantibodies in PNP sera have all proven to be cytoplasmic plaque proteins of the plakin family. These autoantibodies have become indispensable as serologic markers of the disease, but the intracellular localization of the antigens with which they react raises questions about the pathophysiological relevance of these autoantibodies in epidermal blister formation.

We have demonstrated that all 25 well-characterized PNP sera contained IgG autoantibodies that were reactive with 

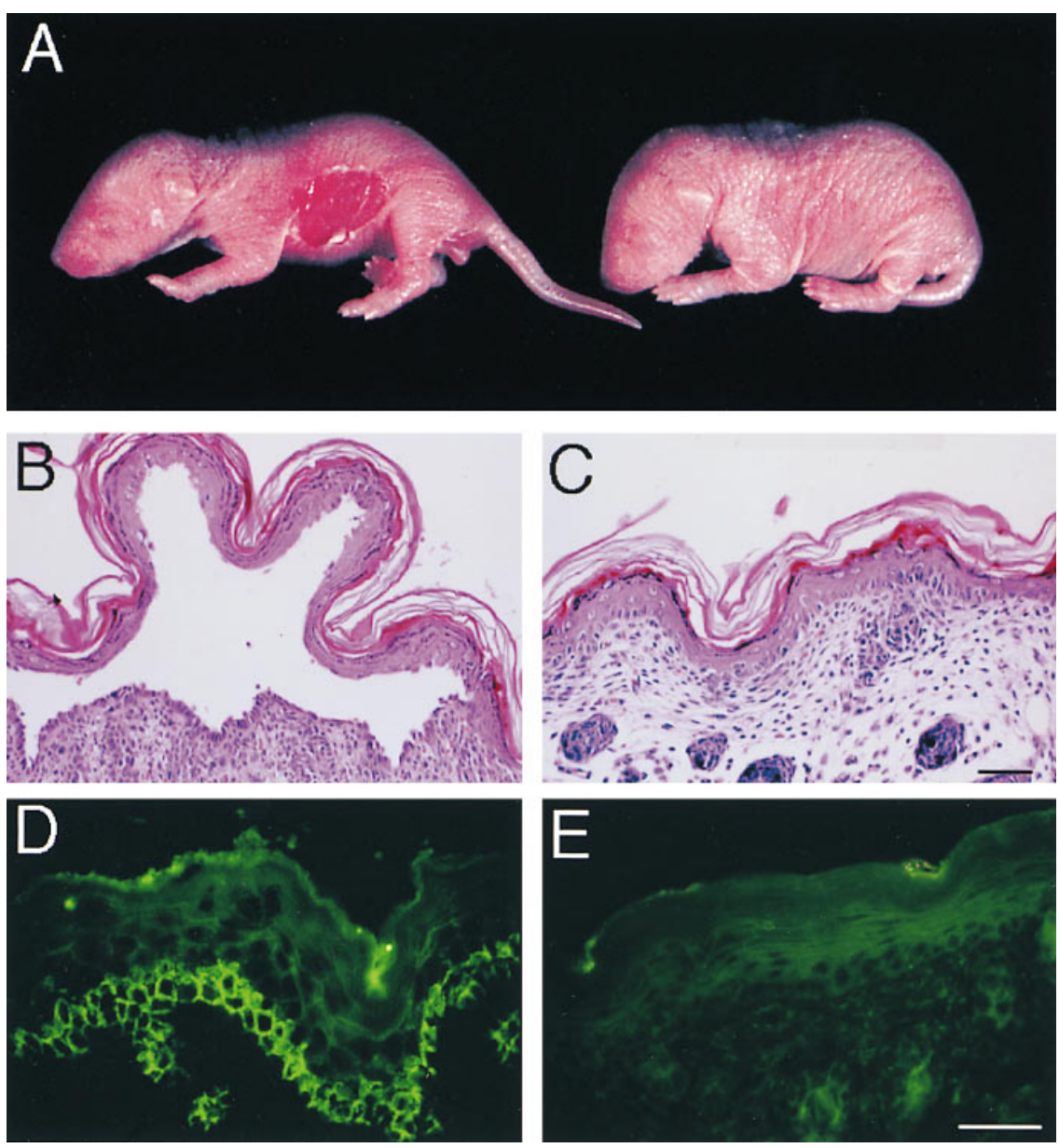

Figure 5. Anti-Dsg3 antibodies affinity purified from PNP sera are pathogenic and cause blister formation in mice. Antibodies were affinity purified on rDsg3-Ig-His or the resin itself from PNP\#1 serum and injected into neonatal mice. The anti-Dsg3-specific antibodies resulted in the formation of gross blisters $(A$, left mouse), with suprabasilar acantholysis in histology $(B)$ and positive human IgG deposition only in the lower epidermis $(D)$. Antibodies eluted from the control resin failed to produce any sign of blister formation after injection into mice ( $A$, right mouse, $B$ ), and no human $\operatorname{IgG}$ was detectable in the skin $(E)$.
Dsg3 by ELISA and that 16 of them also had anti-Dsg1 IgG. Twelve of these PNP sera were further tested, and all were shown to immunoprecipitate rDsg3-His from the culture supernatants of insect cells and the 130-kD Dsg3 from cultured keratinocyte extracts. Immunoadsorption studies showed that the removal of anti-Dsg3 IgG by rDsg3-Ig-His was antigen specific, and this specific immunoadsorption eliminated the ability of PNP sera to induce cutaneous blisters in neonatal mice in vivo. Furthermore, the anti-Dsg3-specific IgG that was affinity purified from PNP sera was proven to be pathogenic and caused gross blisters in neonatal mice. These findings convincingly indicate that Dsg3 is also a key cell surface antigen in PNP and that anti-Dsg3 IgG plays a primary pathogenic role in inducing loss of cell adhesion in keratinocytes and in causing blister formation.

Immunoprecipitation of radiolabeled cultured keratinocyte extracts has been a very reliable method of detecting autoantibodies against the PNP antigen complex (4, 8, 22). However, the $130-\mathrm{kD}$ Dsg3 was not included in the PNP antigen complex as originally described because conventional immunoprecipitation failed to detect it (Fig. 3). A band $\sim 130 \mathrm{kD}$ in size was seen only occasionally by immunoprecipitation with PNP sera $(8,14)$. Our immunoprecipitation-immunoblot assay, which is more sensitive and specific for detection of Dsg3 than conventional immunoprecipitation, clearly demonstrated that PNP sera could immunoprecipitate Dsg3. Therefore, the bands for Dsg3 might have been masked by the more heavily labeled and rapidly developing bands for the plakin family antigens.
PV and PNP share at least two features, which can be explained by the existence of anti-Dsg3 IgG autoantibodies in their sera. The first one is mucous membrane involvement, which is a constant feature of both PV and PNP. The oral mucous membrane is a primary affected site of PV; a recent study showed that Dsg3 is expressed in oral mucous membrane at a higher level than in the epidermis, while Dsg1 is expressed more in the epidermis than in oral mucous membranes (23). This observation, and the clinical appearances of patients with $\mathrm{PV}$, suggests that the more antigen that is present in a tissue, the more damage is caused by autoantibodies. Because essentially all PNP sera contain anti-Dsg3 IgG, the involvement of mucous membrane in PNP patients is well explained by the anti-Dsg3 autoantibodies.

The second common feature is the suprabasilar acantholysis, which is the typical histologic finding in both PV and PNP $(4,24)$. It is clearly established that the suprabasilar acantholysis observed in PV is caused by anti-Dsg3-specific IgG. This is based on the following observations: (a) IgG prepared from PV sera can cause suprabasilar acantholysis without complement or inflammatory cells in a skin organ culture $(25,26) ;(b)$ passive transfer of IgG from PV sera to neonatal mice results in suprabasilar acantholysis (24); (c) immunoadsorption of anti-Dsg3 IgG from PV sera with rDsg3 abolishes blister formation in neonatal mice (16); and (d) Dsg3 is predominantly expressed in basal and parabasal layers of the human epidermis, which is the site of suprabasilar acantholysis (27). Therefore, the finding of anti-Dsg3 IgG in PNP sera now explains 
why suprabasilar acantholysis is also the typical histologic finding in PNP.

Another feature common to PV and PNP and described in this study is the existence of anti-Dsg1 autoantibodies in a subset of patients. It has already been shown that some PV sera contain anti-Dsg1 IgG autoantibodies in addition to anti-Dsg3 IgG (28-30). A recent study with a large number of PV sera demonstrated that 26 out of 46 PV sera (57\%) contained both anti-Dsg3 and anti-Dsg1 IgG (18). In this study, 16 of 25 PNP sera (64\%) were reactive with both Dsg3 and Dsg1, while 13 of $21 \mathrm{PV}$ sera $(62 \%)$ were reactive with both. Thus, the frequency of additional reactivity against Dsg1 is similar in both PV and PNP, suggesting that there may be a common immunopathological mechanism in the break of immunologic tolerance against Dsg.

A more intriguing question is why the clinical presentations of PNP and PV have distinctive differences, despite the common presence of pathogenic anti-Dsg3 IgG autoantibodies. Mucous membrane involvement in PNP is more severe, persistent, and resistant to treatment than that observed in PV. The stomatitis of PNP consists of erosions and ulcerations that affect all surfaces of the oropharynx and characteristically extend onto and involve the vermilion of the lips (5). The cutaneous lesions of PNP are quite variable and more polymorphic than those of PV, and can be morbilliform, lichenoid, or resemble cutaneous lesions of erythema multiforme. The histology of cutaneous lesions in PNP is as varied as the clinical features. In addition to suprabasilar acantholysis, other features include lichenoid changes, vacuolar interface change, or necrosis of individual keratinocytes, which is similar to that observed in erythema multiforme. Furthermore, in PNP a subset of patients has life-threatening pulmonary involvement with acantholytic lesions of bronchial respiratory epithelium. In vivo $\operatorname{IgG}$ autoantibody deposition in bronchial epithelium, where Dsg3 and Dsg1 are not expressed, has been observed in patients with PNP who died of pulmonary failure (31). Such pulmonary disease has never been observed in patients with PV, where lesions are strictly limited to stratified squamous epithelia. At this point, we do not have clear evidence as to what distinct pathophysiologic events are responsible for these differences; however, we can speculate that the existence of autoantibodies against other molecules in the PNP antigen complex may play a role. This may be most relevant in the pulmonary involvement of PNP, as respiratory epithelium does not express Dsg1, Dsg3, or even the 170-kD PNP antigen (32).

With regard to the cutaneous lesions, in PV sera there are no specific autoantibodies other than anti-Dsg3 IgG or antiDsg1 IgG $(16,17,27)$, whereas in PNP, autoantibodies against several members of the plakin family and against the unidentified $170-\mathrm{kD}$ surface protein are consistently present. One could speculate, for example, that anti-Dsg3 IgG initiates the loss of cell adhesion of keratinocytes and causes some damage to the cell membranes. As soon as the membrane is damaged, autoantibodies against the plakin family get into the cell and bind to the target antigens, inhibiting their functions and perhaps precipitating necrosis of keratinocytes. The observation of IgG deposition in the cytoplasm of the affected keratinocytes supports this speculation (Fig. $4 D$ ). There is also some basis for this speculation in a related bullous disease, bullous pemphigoid (33). Rabbits immunized with peptides of the bullous pemphigoid antigen, BPAG1, a member of the plakin family, developed an enhanced inflammatory reaction to ultra- violet $\mathrm{B}$ irradiation, which led to epidermal necrosis and sloughing at some sites.

There is an additional important observation. Mice injected with immunoadsorbed PNP sera, in which all anti-Dsg3 or anti-Dsg1 IgG reactivity was removed, still demonstrated deposition of IgG autoantibodies on the keratinocyte cell surfaces (Fig. $4 E$ ). This observation indicates that there is an unidentified cell surface PNP antigen, which could well be the $170-\mathrm{kD}$ antigen. Autoantibodies against this molecule also might contribute to some features of the human disease that are not easily reproducible during short-term passive transfer experiments in the mice.

Identification of Dsg3 as a target antigen for pathogenic autoantibodies in PNP sera, which induce the loss of cell-cell adhesion of keratinocytes, has provided an important advancement in understanding the pathophysiological mechanism of PNP. This observation has also shed light on the profound similarities and dissimilarities between PV and PNP. However, further extensive studies are needed to clarify the mechanisms responsible for the break of tolerance to these self antigens and to more fully understand the etiology of pemphigus.

\section{Acknowledgments}

We thank Dr. Tadeusz Chorzelski for providing some of the PNP sera used in this study. We also thank Yoshiko Fujii for cell culture and preparation of recombinant baculoproteins, Minae Suzuki for immunofluorescence and immunoblotting, and Kanako Tsutsumi for preparation of histologic paraffin slides. We also thank Kazuhiko Ohya for preparing the reagents for Dsg3 and Dsg1 ELISA.

This work was supported by a Grant-in-Aid for Scientific Research from the Ministry of Education, Science, and Culture of Japan, a grant from the Ministry of Health and Welfare of Japan, research grants from Life Sciences and Medicine, Keio University Medical Science Fund, and Keio Gijuku Academic Development Funds.

\section{References}

1. Stanley, J.R. 1993. Pemphigus. In Dermatology in General Medicine. T.B. Fitzpatrick, A.Z. Eisen, K. Wolff, I.M. Freedberg, and K.F. Austen, editors. McGraw-Hill, New York. 606-614.

2. Amagai, M., V. Klaus-Kovtun, and J.R. Stanley. 1991. Autoantibodies against a novel epithelial cadherin in pemphigus vulgaris, a disease of cell adhesion. Cell. 67:869-877.

3. Amagai, M. 1996. Pemphigus: autoimmunity to epidermal cell adhesion molecules. Adv. Dermatol. 11:319-352.

4. Anhalt, G.J., S. Kim, J.R. Stanley, N.J. Korman, D.A. Jabs, M. Kory, H. Izumi, H. Ratrie, D. Mutasim, A.L. Ariss, and R.S. Labib. 1990. Paraneoplastic pemphigus. An autoimmune mucocutaneous disease associated with neoplasia. N. Engl. J. Med. 323:1729-1735.

5. Anhalt, G.J. 1997. Paraneoplastic pemphigus. Adv. Dermatol. 12:77-97.

6. Oursler, J.R., R.S. Labib, A.L. Ariss, T. Burke, E.J. O'Keefe, and G.J. Anhalt. 1992. Human autoantibodies against desmoplakins in paraneoplastic pemphigus. J. Clin. Invest. 89:1775-1782.

7. Stanley, J.R. 1993. Cell adhesion molecules as targets of autoantibodies in pemphigus and pemphigoid, bullous diseases due to defective epidermal cell adhesion. Adv. Immunol. 53:291-325.

8. Hashimoto, T., M. Amagai, K. Watanabe, T.P. Chorzelski, B.S. Bhogal, M.M. Black, H.P. Stevens, D.M. Boorsma, N.J. Korman, and T. Nishikawa. 1995. Characterization of paraneoplastic pemphigus autoantigens by immunoblot analysis. J. Invest. Dermatol. 104:829-834.

9. Kim, S.C., Y.D. Kwon, I.J. Lee, S.N. Chang, and T.G. Lee. 1997. cDNA cloning of the $210-\mathrm{kDa}$ paraneoplastic pemphigus antigen reveals that envoplakin is a component of the antigen complex. J. Invest. Dermatol. 109:365369 .

10. Ruhrberg, C., M.A. Hajibagheri, M. Simon, T.P. Dooley, and F.M. Watt. 1996. Envoplakin, a novel precursor of the cornified envelope that has homology to desmoplakin. J. Cell. Biol. 134:715-729.

11. Aho, S., W.H.I. McLean, M. Mahoney, J.R. Stanley, and J. Uitto. 1998. Human periplakin: cDNA and genomic cloning, chromosomal mapping, mRNA expression, and a paraneoplastic antigen. J. Invest. Dermatol. 110:473a. 
(Abstr.)

12. Ruhrberg, C., M.A.N. Hajibagheri, D.A.D. Parry, and F.M. Watt. 1997. Periplakin, a novel component of cornified envelopes and desmosomes that belongs to the plakin family and forms complexes with envoplakin. J. Cell. Biol. 139:1835-1849.

13. Joly, P., E. Thomine, D. Gilbert, S. Verdier, A. Delpech, C. Prost, C. Lebbe, P. Lauret, and F. Tron. 1994. Overlapping distribution of autoantibody specificities in paraneoplastic pemphigus and pemphigus vulgaris. J. Invest. Dermatol. 103:65-72.

14. Hashimoto, T., M. Amagai, N. Wang, T. Nishikawa, T. Karashima, O. Moria, S. Jablonska, and T.P. Chorzelski. 1998. Novel non-radioisotope immunoprecipitation studies indicate involvement of pemphigus vulgaris antigen in paraneoplastic pemphigus. J. Dermatol. Sci. 17:132-139.

15. Ruhrberg, C., and F.M. Watt. 1997. The plakin family: versatile organizers of cytoskeletal architecture. Curr. Opin. Genet. Dev. 7:392-397.

16. Amagai, M., T. Hashimoto, N. Shimizu, and T. Nishikawa. 1994. Absorption of pathogenic autoantibodies by the extracellular domain of pemphigus vulgaris antigen (Dsg3) produced by baculovirus. J. Clin. Invest. 94:59-67.

17. Amagai, M., T. Hashimoto, K.J. Green, N. Shimizu, and T. Nishikawa. 1995. Antigen-specific immunoadsorption of pathogenic autoantibodies in pemphigus foliaceus. J. Invest. Dermatol. 104:895-901.

18. Ishii, K., M. Amagai, R.P. Hall, T. Hashimoto, A. Takayanagi, S. Gamou, N. Shimizu, and T. Nishikawa. 1997. Characterization of autoantibodies in pemphigus using antigen-specific ELISAs with baculovirus expressed recombinant desmogleins. J. Immunol. 159:2010-2017.

19. Hashimoto, T., M.M. Ogawa, A. Konohana, and T. Nishikawa. 1990. Detection of pemphigus vulgaris and pemphigus foliaceus antigens by immunoblot analysis using different antigen sources. J. Invest. Dermatol. 94:327-331.

20. Tsukamoto, T. 1989. Establishment and characterization of a cell line (KU8) from squamous cell carcinoma of the penis. Keio J. Med. 38:277-293.

21. Liu, A.Y., R. Valenzuela, T.N. Helm, C. Camisa, A.L. Melton, and W.F. Bergfeld. 1993. Indirect immunofluorescence on rat bladder transitional epithelium: a test with high specificity for paraneoplastic pemphigus. J. Am. Acad. Dermatol. 28:696-699.

22. Helou, J., J. Allbritton, and G.J. Anhalt. 1995. Accuracy of indirect immunofluorescence testing in the diagnosis of paraneoplastic pemphigus. J. Am. Acad. Dermatol. 32:441-447.
23. Shirakata, Y., M. Amagai, Y. Hanakawa, T. Nishikawa, and K. Hashimoto. 1998. Lack of mucosal involvement in pemphigus foliaceus may be due to low expression of desmoglein1. J. Invest. Dermatol. 110:76-78.

24. Anhalt, G.J., R.S. Labib, J.J. Voorhees, T.F. Beals, and L.A. Diaz. 1982. Induction of pemphigus in neonatal mice by passive transfer of IgG from patients with the disease. N. Engl. J. Med. 306:1189-1196.

25. Schiltz, J.R., and B. Michel. 1976. Production of epidermal acantholysis in normal human skin in vitro by the $\mathrm{IgG}$ fraction from pemphigus serum. J. Invest. Dermatol. 67:254-260.

26. Hashimoto, K., K.M. Shafran, P.S. Webber, G.S. Lazarus, and K.H. Singer. 1983. Anti-cell surface pemphigus autoantibody stimulates plasminogen activator activity of human epidermal cells. J. Exp. Med. 157:259-272

27. Amagai, M., P.J. Koch, T. Nishikawa, and J.R. Stanley. 1996. Pemphigus vulgaris antigen (Desmoglein 3 ) is localized in the lower epidermis, the site of blister formation in patients. J. Invest. Dermatol. 106:351-355.

28. Eyre, R.W., and J.R. Stanley. 1988. Identification of pemphigus vulgaris antigen extracted from normal human epidermis and comparison with pemphigus foliaceus antigen. J. Clin. Invest. 81:807-812.

29. Emery, D.J., L.A. Diaz, J.A. Fairley, A. Lopez, A.F. Taylor, and G.J. Giudice. 1995. Pemphigus foliaceus and pemphigus vulgaris autoantibodies react with the extracellular domain of desmoglein-1. J. Invest. Dermatol. 104:323328

30. Kowalczyk, A.P., J.E. Anderson, J.E. Borgwardt, T. Hashimoto, J.R Stanley, and K.J. Green. 1995. Pemphigus sera recognize conformationally sensitive epitopes in the amino-terminal region of desmoglein-1 (Dsg1). J. Invest. Dermatol. 105:147-152.

31. Fullerton, S.H., D.T. Woodley, B.R. Smoller, and G.J. Anhalt. 1992. Paraneoplastic pemphigus with autoantibody deposition in bronchial epithelium after autologous bone marrow transplantation. JAMA. 267:1500-1502.

32. Nousari, H.C., H.A. Wotczak, and G.J. Anhalt. 1997. Autoantibodymediated acantholysis of respiratory epithelium in paraneoplastic pemphigus. $J$. Invest. Dermatol. 108:641a. (Abstr.)

33. Hall, R.P., J.C. Murray, M.M. McCord, M.J. Rico, and R.D. Streilein. 1993. Rabbits immunized with a peptide encoded for by the 230-kD bullous pemphigoid antigen cDNA develop an enhanced inflammatory response to UVB irradiation: a potential animal model for bullous pemphigoid. J. Invest. Dermatol. 101:9-14. 